\title{
Ulcerative Colitis: A Genetically Heterogeneous Disorder Defined by Genetic (HLA Class II) and Subclinical (Antineutrophil Cytoplasmic Antibodies) Markers
}

\author{
Huiying Yang, Jerome I. Rotter, Hiroo Toyoda, Carol Landers, Dolly Tyan, Colleen K. McElree, and Stephan R. Targan \\ Divisions of Medical Genetics and Gastroenterology, Departments of Medicine and Pediatrics, The Inflammatory Bowel Disease Center, \\ Cedars-Sinai Medical Center, and the University of California at Los Angeles School of Medicine, Los Angeles, California 90048
}

\begin{abstract}
Newly described distinct associations of HLA class II genes with ulcerative colitis (UC) (DR2) and Crohn's disease (CD) (DR1 / DQ5) provide strong evidence for genetic heterogeneity of susceptibility between these two forms of inflammatory bowel disease. A familial distribution of antineutrophil cytoplasmic antibodies (ANCAs, a subclinical marker of $U C$ ) in UC families has further implied the existence of heterogeneity within UC.

To test the hypothesis that the heterogeneity within UC indicated by ANCAs has a genetic basis that resides within the HLA region, we studied $89 \mathrm{UC}$ cases and an ethnically matched control group $(n=50)$. Serological and molecular typing techniques were applied to define HLA class II genes (DR, DQ). ANCAs were detected using an enzyme-linked immunosorbent assay, and positive values were confirmed by indirect immunofluorescence.

We observed that ANCA-positive UC patients $(n=70)$ had a significantly increased frequency of DR2 compared with ANCA-negative controls $(n=46)(44 \%$ vs $22 \%, P=0.01)$. In contrast, the frequency of DR2 in ANCA-negative UC cases (21\%) was virtually identical to that in controls $(22 \%, P=0.9)$. Furthermore, the ANCA-negative UC patients had an increase in the DR4 allele compared with ANCA-positive UC ( $P$ $=0.004)$.

Thus, with the combination of a subclinical marker (ANCAs) and molecular genetic markers, genetic heterogeneity has been demonstrated within UC: ANCA-positive UC associated with DR2, and ANCA-negative UC likely associated with DR4. (J. Clin. Invest. 1993. 92:1080-1084.) Key words: inflammatory bowel disease $\bullet$ HLA association • antibodies
\end{abstract}

\section{Introduction}

Ulcerative colitis (UC) ${ }^{1}$ and Crohn's disease (CD) are classified as the chronic idiopathic inflammatory bowel diseases

Address correspondence and reprint requests to Huiying Yang, M.D., Ph.D., Division of Medical Genetics, SSB-378, Cedars-Sinai Medical Center, 8723 Alden Drive, Los Angeles, CA 90048.

Received for publication 30 November 1992 and in revised form 2 April 1993.

1. Abbreviations used in this paper: ANCA, antineutrophil cytoplasmic antibody; CD, Crohn's Disease; IBD, inflammatory bowel disease;

J. Clin. Invest.

(C) The American Society for Clinical Investigation, Inc.

0021-9738/93/08/1080/05 \$2.00

Volume 92, August 1993, 1080-1084
(IBD). The etiology and pathogenesis of these disorders has not yet been elucidated. Important clues have come from genetic epidemiologic studies of IBD, which have demonstrated that there is familial aggregation, an increased monozygotic twin concordance, and lack of increased risk in spouses, suggesting the importance of genetic factors in the etiology of these disorders (1).

There is an increasing body of evidence suggesting genetic heterogeneity within IBD (1-4). If the heterogeneity model is indeed the appropriate explanation, an understanding of the etiologies of IBD will only be possible by subdividing these disorders into more etiologically homogeneous groups on the basis of both physiologic defects and genetic marker associations.

HLA class II genes are primary candidate genes for the genetic study of IBD because they are $(a)$ crucial in antigen recognition; $(b)$ fundamental to the immune response; and $(c)$ associated with various autoimmune diseases. In a previous study, we have observed distinct and different associations of HLA class II genes with UC and with CD (4). UC was positively associated with the HLA DR2 allele and negatively associated with DR4 and DR6, while CD was positively associated with the combination of DR 1 and DQ5 alleles. These data indicate that the DR and DQ molecules separate $U C$ and CD on genetic grounds, and that the susceptibility contributed by the HLA class II genes is quite different for the two disorders.

Antineutrophil cytoplasmic antibodies (ANCAs) were first observed as an important marker in Wegener's granulomatosis in 1985 (5). In 1990, a distinct subset of ANCA was found to be associated with UC (6). The prevalence of positive ANCA in patients with UC ranges from 50 to $86 \%(3,6-9)$. This UC-associated ANCA has a perinuclear immunofluorescence binding pattern that is different from other ANCAs, such as the granular cytoplasmic pattern in Wegener's and the nuclear staining in systemic lupus erythematosus. There has not been as yet any detectable difference in the level of ANCA binding between UC patients with rectosigmoid disease and those with pancolitis, or between patients with active disease and those with inactive colitis $(6,8)$. Importantly, ANCAs have also been found in patients with UC after colectomy. This antibody is highly specific for ulcerative colitis compared with other forms of colitis, including that of Crohn's colitis (7-9). We have also found that ANCAs have an increased frequency among the clinically healthy relatives of UC patients compared with environmental and ethnically matched controls (3).

IDDM, insulin-dependent diabetes mellitus; PBL, peripheral blood leukocytes; UC, ulcerative colitis. 
These cumulative observations, constancy, specificity, and increased prevalence in unaffected relatives, suggest that ANCAs are not simply an epiphenomenon related to active colonic inflammation. These data suggest that the presence of ANCAs in patients with UC may reflect a fundamental disturbance of immune regulation.

The fact that not all UC patients have ANCAs suggests the possibility of heterogeneity within UC. This UC-specific antibody has a familial distribution; i.e., the relatives of ANCA-positive UC patients have an increased prevalence of presence of ANCA compared with the relatives of ANCA-negative UC patients (3). The reason for such a familial distribution of ANCA has not been elucidated. However, since UC is associated with specific HLA class II alleles (4), it is possible that these ANCA differences have a genetic basis that resides within the HLA complex. In the present study, we have tested that hypothesis by examining the associations with HLA class II genes as a function of ANCA status. The results suggest that expression of this antibody in UC is controlled by genes closely linked to the HLA class II gene region and that ANCA-positive and ANCAnegative UC are genetically distinct groups.

\section{Methods}

\section{Study subjects}

Patients. We ascertained a total of 89 UC patients from the clinical IBD programs at UCLA and Cedars-Sinai. The study protocols were approved by each institution's Human Subject Review Committee. The diagnosis of UC was documented by conventional endoscopic, histological, and clinical criteria. The UC patients studied did not have multiple sclerosis, systemic lupus erythematosus, or other recognized autoimmune diseases.

Controls. To select an ethnically, socioeconomically matched control group for the association study, we ascertained 50 controls from the spouses or acquaintances of the patients. An individual was used as control only if he/she did not have inflammatory bowel disease, multiple sclerosis, systemic lupus erythematosus, or other recognized autoimmune diseases. The distribution of age, gender, and ethnicity (Jewish/non-Jewish) were comparable between patients and controls. All patients and controls analyzed in the present paper are Caucasians.

\section{Detection of ANCAS by ELISA}

The development and validation of the fixed ANCA ELISA has been described in detail elsewhere (6). In brief, purified neutrophils from a single normal individual served as the source of antigen and were isolated by Ficoll-Hypaque (specific gravity 1.080) density centrifugation followed by dextran sedimentation of the cell pellet (10). Microtiter wells (Immulon 2; Dynatech Laboratories, Alexandria, VA) were coated with a monolayer of neutrophils by the addition of $100 \mu \mathrm{l} /$ well of Hanks' balanced buffered salt solution containing 250,000 neutrophils. After the cells had settled and spread for $30 \mathrm{~min}$ at room temperature, the microtiter plates were centrifuged at $1,000 \mathrm{rpm}(300 \mathrm{~g})$ for 5 min, the supernatant was aspirated from the wells, and the plates were air dried. The cells were fixed with $100 \%$ methanol for $10 \mathrm{~min}$, after which the plates were air dried and stored at $-20^{\circ} \mathrm{C}$. For use, the plates were brought to room temperature, and $150 \mu \mathrm{l}$ of $0.25 \%$ BSA in PBS was added to each microtiter well for $1 \mathrm{~h}$ to block nonspecific binding. The blocking material was discarded, and $100 \mu \mathrm{l}$ of test serum diluted in BSA/PBS (or BSA/PBS alone for blank wells) was added. To standardize the assay, a positive pool of sera from six individuals with ulcerative colitis (three with very high and three with intermediate levels of ANCA) was used. This was included on each microtiter plate at a dilution of 1:100. The plates were incubated for $1 \mathrm{~h}$ at room temperature in a humidified box. They were then washed three times with $0.05 \%$ Tween 20 in PBS (PBS/Tween), and $100 \mu \mathrm{l} /$ well of a 1:750 dilution of alkaline phosphatase coupled goat anti-human gamma chain specific antibody (Tago, Inc., Burlingame, CA) in BSA/PBS was added for $1 \mathrm{~h}$. This antibody was discarded, and the wells were washed three times with PBS/Tween and four times with $0.05 \mathrm{M}$ Tris base in $0.9 \mathrm{M} \mathrm{NaCl}, \mathrm{pH} 7.5$. Substrate solution $(1.5 \mathrm{mg} / \mathrm{ml}$ disodium $p$-nitrophenol phosphate in $0.01 \mathrm{M}$ Tris base, $0.0025 \mathrm{M} \mathrm{MgCl}_{2}, \mathrm{pH} 8.6,100$ $\mu \mathrm{l} /$ well) was added, and color development was allowed to proceed until absorbance at $405 \mathrm{~nm}$ in the positive control wells was 0.8-1.0 optical density units greater than in blank wells. Results for test sera were expressed in terms of percent of positive control after correction for background as measured in blank wells.

\section{Confirmation of positive ELISA values by indirect immunofluorescence}

Sera that exhibited levels of binding $>2$ SD above the mean level for the normal laboratory controls at a dilution of $1 / 100$ in the ELISA were regarded as being positive and were also examined by indirect immunofluorescence to confirm that the antibody binding was cell-specific. Cytocentrifuged smears with $\sim 100,000$ neutrophils/slide were fixed in $100 \%$ methanol at room temperature and the coded sera were tested at a dilution of 1:20 and stained with fluorescein-labeled $F\left(a b^{\prime}\right)_{2}$ gamma chain-specific antibody as described before (6).

\section{DNA isolation and genomic blotting}

Genomic DNA was isolated from all subjects. These DNA samples were from either peripheral blood leukocytes (PBL) or EBV-transformed cell lines. High molecular weight genomic DNA was prepared from PBL according to the methods described previously with minor modifications $(11,12)$. Briefly, PBL were isolated from $5 \mathrm{ml}$ blood collected into an EDTA tube and were resuspended in $400 \mu \mathrm{l}$ of buffer (Tris- $\mathrm{HCl}, \mathrm{pH} 8.0,10 \mathrm{mM}$ EDTA, pH $8.0,150 \mathrm{mM} \mathrm{NaCl}$ ) with $1 \%$ SDS and proteinase $\mathrm{K}(500 \mu \mathrm{g} / \mathrm{ml}$, Boeringer Mannheim Biochemicals, Indianapolis, IN). The reaction solution was incubated overnight at $60^{\circ} \mathrm{C}$, followed by phenol-chloroform extraction. The DNA was precipitated with $100 \%$ cold ethanol and resuspended in buffer $(1.0$ mM Tris-HCl, pH 8.0, 0.1 mM EDTA, pH 8.0). About 50-100 $\mu \mathrm{g}$ of DNA $/ 5 \mathrm{ml} \mathrm{PBL}$ was isolated by this protocol. DNA samples $(10 \mu \mathrm{g})$ were digested with TaqI restriction enzyme, electrophoresed, and transferred onto MSI nylon membrane (Fisher Scientific Co., Pittsburgh, PA) using the Southern blot technique (13). The cDNA probes were labeled by the random-primer method (14), and hybridization was carried out using previously described methods $(11,12)$. The DRbeta probe was prepared by digestion of DR-beta cDNA clone (15) with PstI, generating a 520-bp fragment. The DQ-beta probe was prepared by digestion of a DQ-beta cDNA clone (pII-beta-1) (16) with AvaI, generating a 630-bp fragment. The membranes were hybridized at $65^{\circ} \mathrm{C}$ for $12 \mathrm{~h}$, and subsequently washed as follows: $2 \times$ SSC- $-1 \%$ SDS at $65^{\circ} \mathrm{C}$ for $5 \mathrm{~min}$ twice, followed by $0.2 \times$ SSC -0.1 SDS at $65^{\circ} \mathrm{C}$ for $5 \mathrm{~min}$ twice. Then the membranes were exposed with $\mathrm{x}$-ray film (XAR-5; Kodak Corp., Rochester, NY) using one intensifying screen (Du Pont Pharmaceuticals, Inc., Wilmington, DE) at $-70^{\circ} \mathrm{C}$.

\section{HLA typing}

The double immunofluorescence procedure was used to perform HLA serologic typing for class II antigens (17). Typing for the class II antigens was performed using internationally exchanged and local alloantisera developed in the HLA laboratory at Cedars-Sinai Medical Center and One Lambda (Los Angeles, CA) commercial DR trays.

DR-DQ genotyping by RFLP was carried out to detect various DRand DQ-beta alleles (11). This method was used to assign alleles to individuals when serologic HLA typing could not distinguish the alleles.

\section{Statistical analysis}

The significance of any associations of HLA DR and DQ alleles with subsets of UC indicated by ANCA status was assessed using a chisquare test or Fisher's exact probability test when appropriate (18). Only four of the controls were ANCA positive, which was too small for 
Table I. Characteristics of ANCA-Positive and ANCA-Negative Groups

\begin{tabular}{|c|c|c|c|c|}
\hline & $\begin{array}{l}\text { ANCA-pos* } \\
\text { UC }\end{array}$ & $\begin{array}{l}\text { ANCA-neg }{ }^{\ddagger} \\
\text { UC }\end{array}$ & $\begin{array}{l}\text { ANCA-pos* } \\
\text { Control }\end{array}$ & $\begin{array}{l}\text { ANCA-neg }^{\ddagger} \\
\text { Control }\end{array}$ \\
\hline$n$ & 70 & 19 & 4 & 46 \\
\hline Male/female & $33 / 37$ & $8 / 11$ & $2 / 2$ & $22 / 24$ \\
\hline Percent Jewish & 44.8 & 27.8 & 50.0 & 30.4 \\
\hline \multicolumn{5}{|l|}{ Age (yr) } \\
\hline Median \pm SD & $38 \pm 2.0$ & $38 \pm 4.6$ & $38.5 \pm 8.1$ & $40 \pm 3.2$ \\
\hline Mean \pm SD & $40.2 \pm 1.6$ & $43.6 \pm 3.7$ & $41.3 \pm 6.7$ & $42.3 \pm 2.3$ \\
\hline Range & $20-82$ & $25-83$ & $30-58$ & $21-77$ \\
\hline \multicolumn{5}{|c|}{ Age at onset of $U C(\mathrm{yr})$} \\
\hline Median \pm SD & $27.0 \pm 1.7$ & $27.0 \pm 3.2$ & & \\
\hline Mean \pm SD & $29.5 \pm 1.7$ & $29.3 \pm 3.2$ & & \\
\hline Range & $3-66$ & $10-48$ & & \\
\hline \multicolumn{5}{|c|}{ Disease duration (yr) } \\
\hline Median \pm SD & $7.5 \pm 1.4$ & $7.0 \pm 5.5$ & & \\
\hline Mean \pm SD & $10.5 \pm 1.1$ & $13.5 \pm 4.1$ & & \\
\hline Range & $1-44$ & $1-68$ & & \\
\hline
\end{tabular}

* ANCA-positive, ${ }^{\ddagger}$ ANCA-negative.

meaningful statistical comparisons. Thus, all comparisons were done between the ANCA-positive UC patients, the ANCA-negative UC patients, and the ANCA-negative controls. The Mental-Haenszel test (19) was performed for stratified analyses.

As the hypothesis regarding DR2 was generated from previous association studies (see ref. 4 for all relevant reports), this was considered to be a specific hypothesis and was tested individually. Corrections for multiple comparisons were made for the tests on the remaining alleles, which included six additional DR alleles (DR1, DR3, DR4, DR5, DR6, and DR7), and five DQ alleles (DQ2, DQ5, DQ6, DQ7, and DQ8). Those alleles, DR8, DR9, and DR10, found to be extremely infrequent in both cases and controls (ranging from 0 to 2) were not individually analyzed. When corrected for the number of antigens tested, the $P$ value is designated as $P_{\text {corr }}$.

\section{Results}

Demographic characteristics of UC patients and controls. Among the UC patients, 78.7\% (70/89) were ANCA-positive. The demographic characteristics of the patients and controls, stratified by ANCA status, are summarized in Table 1 . The sex ratio, age, age of onset, and disease duration did not differ between groups. In both the UC and control groups, it appeared that the proportion of individuals whose ancestry was Jewish was greater in the ANCA-positive group than that in the ANCA-negative group, though this did not attain statistical significance $(P=0.066$ for the pooled data $)$. In the subsequent analyses of DR and DQ associations as a function of ANCA status, we first analyzed the Jewish and non-Jewish subjects separately to identify any potential ethnic difference in such associations. Our analyses indicated that there was no significant difference between Jews and non-Jews in the HLA class II gene associations with UC or with ANCA. Thus, we report the results from the pooled sample.

HLA DR2 in ANCA-positive and ANCA-negative UC patients compared with controls. To examine any relation between DR2 and ANCA, we compared the frequency of the DR2 allele in ANCA-positive UC and in ANCA-negative UC with ANCA-negative controls. As discussed under Methods, there were only four ANCA-positive controls, which was sim- ply too small a group to include in the comparisons. ANCApositive UC had a significantly increased frequency of DR2 compared with ANCA-negative controls (44.3\% vs $21.7 \%, P$ $=0.013$ ) (Table II and Fig. 1). In contrast, not only was there no difference, but there was virtual identity in the DR2 frequency between ANCA-negative UC and ANCA-negative controls ( 21.1 vs $21.7 \%, P=0.95)$. Within UC patients, the DR2 frequency in ANCA-positive individuals was more than twofold greater compared to that in ANCA-negative individuals (44.3 vs $21.1 \%, P=0.066$ ) (Fig. 1).

HLA DR4 and DR6 in ANCA-negative UC patients and controls. We have previously reported that frequencies of the HLA DR4 and DR6 antigens were decreased in patients with UC compared with controls (4). Thus, we investigated whether these associations were also a function of ANCA status. ANCA-positive UC had a decreased frequency of DR4

Table II. Frequencies of Specified HLA-DR and $D Q$ Alleles in ANCA-Positive UC, ANCA-Negative UC, and ANCA-Negative Controls

\begin{tabular}{lccc}
\hline & $\begin{array}{c}\text { ANCA-positive } \\
\text { UC } \\
n=70 \\
(\%)\end{array}$ & $\begin{array}{c}\text { ANCA-negative } \\
\text { UC } \\
n=19 \\
(\%)\end{array}$ & $\begin{array}{c}\text { ANCA-negative } \\
\text { control } \\
n=46 \\
(\%)\end{array}$ \\
\hline DR1 & 21.4 & 31.6 & 15.2 \\
DR2 & $44.3^{\prime \prime}$ & 21.1 & 21.7 \\
DR4 & $12.9^{*}$ & 42.1 & 19.6 \\
DR6 & $21.4^{8}$ & 26.3 & 41.3 \\
DQ5 & 31.4 & 47.4 & 26.1 \\
DQ7 & 48.6 & 26.3 & 30.4 \\
DQ8 & 20.0 & 36.8 & 21.7 \\
DR1/DQ5 & 17.1 & 26.3 & 13.0 \\
DR4/DQ7 & 5.7 & 5.3 & 8.7 \\
DR4/DQ8 & $10.0^{\ddagger}$ & $31.6^{8}$ & 10.9 \\
& & & \\
\hline
\end{tabular}

* $P=0.004,{ }^{\ddagger} P=0.018$, when compared between ANCA-positive UC and ANCA-negative UC.

${ }^{\S} P<0.05$, " $P=0.013$, when compared with ANCA-negative controls. 


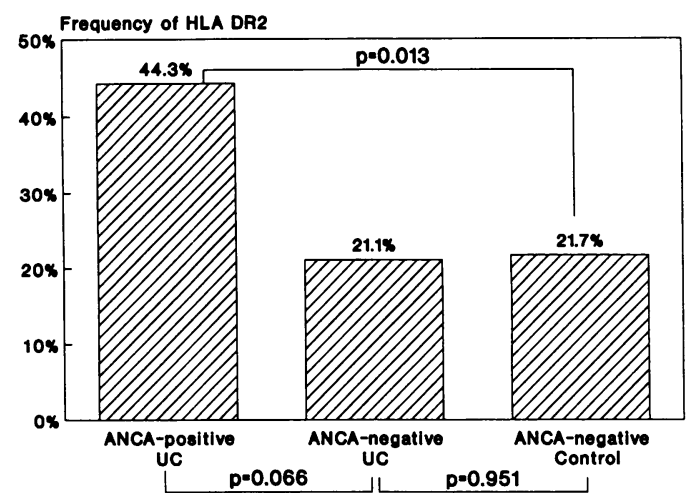

Figure 1. HLA-DR2 allele frequency in ANCA-positive UC and ANCA-negative UC patients, compared with ANCA-negative controls. ANCA-positive UC probands exhibited an increased frequency of DR2 $(P=0.013)$, while ANCA-negative UC patients had the identical frequency of DR2 as controls.

( 12.9 vs $19.6 \%$ ) and DR6 (21.4 vs $41.3 \%$ ) compared with ANCA-negative controls. This was seen in our previous study of all combined UC patients. However, in contrast, the ANCAnegative UC group had an increased frequency of DR4 (42.1 $\%)$. When DR4 was compared within UC, results showed that ANCA-positive UC had a statistically significant decreased DR4 frequency compared with ANCA-negative UC (12.9 vs $42.1 \%, P=0.004, P_{\text {corr }}=0.024$ ) (Fig. 2). To examine the possibility of haplotype contributions, we tested these associations for the combination of DR4/DQ7 or DR4/DQ8. The data clearly showed that it was the combination of DR4/DQ8 that associated with ANCA-negative UC $(P=0.043)$, not the combination of DR4/DQ7 $(P=0.637)$ (Table II). To further examine this relationship, we stratified on DR4 to examine the effect of DQ8, and on DQ8 to examine the effect of DR4. This stratified analysis (Mental-Haenszel test) suggested that the association effect is stronger with the DR locus (data not shown). The overall effect of DR6 was different. There seemed to be no differential association with DR6 between ANCA-negative UC and ANCA-positive UC, with both exhibiting a reduced frequency compared to controls (Table II).

$H L A D R I$ and DQ5 in ANCA-negative UC patients and controls. To further investigate the HLA class II gene associations with ANCA-negative UC, we also examined DR1 and

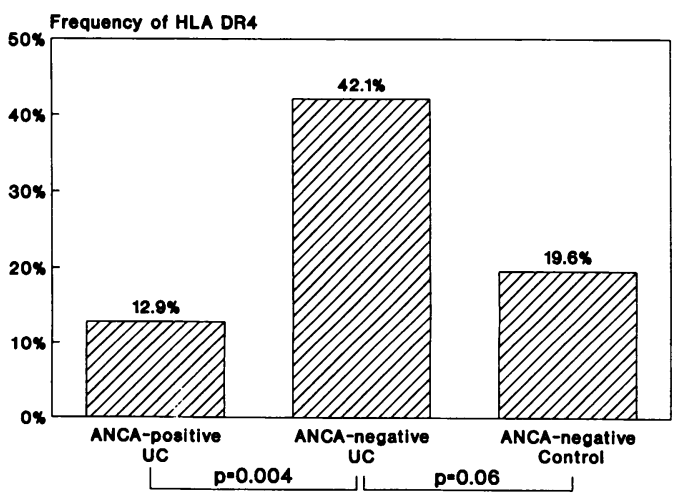

Figure 2. HLA-DR4 allele frequency in ANCA-positive UC and ANCA-negative UC patients, compared with ANCA-negative controls. ANCA-negative UC probands demonstrated an increased frequency of DR4 compared with both ANCA-positive UC patients $(P=0.004)$ and ANCA-negative controls $(P=0.06)$.
DQ5 in the ANCA-negative cases, since these two alleles were found to be associated with Crohn's disease $(4,20)$. Both DR1, DQ5, and the combination of DR1/DQ5 showed a twofold increased frequency in ANCA-negative UC patients compared with ANCA-negative controls (DR1: 31.6 vs $15.2 \%$; DQ5: 47.4 vs $26.1 \%$; DR1/DQ5: 26.3 vs $13.0 \%$ ) (Table II), although the differences did not reach statistical significance ( $P$ $=0.13,0.095$, and 0.194 for DR1, DQ5, and DR1/DQ5, respectively). In addition, the frequencies of DR $1, \mathrm{DQ} 5$, and the combination of DR1/DQ5 in ANCA-negative UC were very similar in magnitude when contrasted to those previously reported in Crohn's (4).

Besides DR1, DR2, DR4, DR6, DQ5, DQ7, and DQ8, we have also examined other HLA class II DR and DQ alleles, including DR3, DR5, DR7, DQ2, and DQ6. None of these alleles showed associations with either ANCA-positive UC or ANCA-negative UC.

\section{Discussion}

In this study, we have investigated the hypothesis that the heterogeneity within UC indicated by the presence or absence of ANCA has a genetic basis. Our data demonstrate that ANCApositive UC and ANCA-negative UC are genetically distinct subgroups of UC: ANCA-positive UC is associated with DR2, while ANCA-negative UC is not. Thus, the positive association of UC with DR2 observed in previous studies appears to be contributed by ANCA-positive UC patients.

It has been noted that the frequency of positivity of ANCA in UC patients varies from population to population. For example, it was $~ 50 \%$ in France (21) and England (8), and $~ 70 \%$ in most U.S. Caucasian patients $(3,6,7)$. Such different prevalences of ANCA in different UC populations may well be determined by the genetic differences in UC susceptibility genes presumably linked to the HLA class II genes in these UC populations. Further studies across populations are needed to characterize the underlying mechanism of population differences in the frequency of ANCA.

Further genetic differences between ANCA-positive UC and ANCA-negative UC were also suggested by the data from the DR4, DR1, and DQ5 alleles. Most prominently, there was a large difference in DR4 frequency between the ANCA-negative and ANCA-positive UC (42.1 vs $12.9 \%, P=0.004)$. It could be argued that the observation of an increased frequency of DR4 in a UC subgroup is an unexpected finding, and would need to be corrected for the number of DR antigens examined, in this case six. The comparison still remains statistically significant after such correction (adjusted $P_{\text {corr }}=0.024$ ). These data suggest that associations (positive/negative) between DR4 and UC taken as one group will be dependent on the ANCA status of the individuals tested. Additional studies with large samples of ANCA phenotyped patients will lead to a better characterization of the DR4 associations with UC.

The DR4 associated with DQ8 seems to be primarily responsible for the ANCA-negative UC susceptibility, rather than the DQ7 associated DR4. A similar DR4/DQ8 association has been observed in another autoimmune disease, insulin dependent diabetes mellitus (IDDM) (22). Furthermore, a large patient series indicated that UC (but not $\mathrm{CD}$ ) is associated with a number of organ specific autoimmune disorders (23), including thyroid disorders, autoimmune hemolytic anemia, IDDM, rheumatoid arthritis, and systemic lupus erythematosus; and some of these autoimmune diseases are asso- 
ciated with DR4; e.g., IDDM and rheumatoid arthritis (24). Likewise, another DR2-associated disease, multiple sclerosis (24), has been found to be clinically associated with IBD (25), particularly with UC (26). These data suggest that both forms of UC, ANCA-positive and ANCA-negative, have an HLAlinked basis, but that it is very different for these two forms of UC: the former is associated with DR2, whereas the latter is associated with DR4. What may be emerging is that in disease susceptibility, and possibly in disease pathogenesis, DR2 associated ANCA-positive UC may overlap with multiple sclerosis, and DR4 associated ANCA-negative UC may overlap with very different autoimmune disorders.

In previous studies by ourselves and others $(4,20)$, DR 1 and DQ5 were found to be positively associated with Crohn's disease, but no association was observed with UC considered as one entity. Of interest, such associations did appear among the ANCA-negative UC patients in this study, although they did not attain statistical significance. The frequencies of these alleles in the ANCA-negative UC patients were very similar in magnitude when contrasted to those previously reported in Crohn's (4) (ANCA-negative UC vs CD-DR1: 31.6 vs $28.4 \%$; DQ5: 47.4 vs $41.1 \%$; DR1 /DQ5: 26.3 vs $27.4 \%$ ). Clinically, UC and CD can be distinguished in most cases by the course of the patient's illness, sites of bowel involvement, together with a combination of laboratory, radiologic, endoscopic, and histologic findings. Genetically, UC and CD may overlap, since the concurrence of both UC and CD in the same family is far greater than that which would be expected by chance alone ( 1 , $2,27,28$ ). Yet UC and CD seem to have entirely different HLA class II associations: DR2 with UC, DR 1 / DQ5 with CD $(4,20)$. The observations in this study, if confirmed, suggest one potential explanation that can resolve these seemingly contradictory observations. The overlap may be caused by ANCAnegative UC (or a subset of ANCA-negative UC) sharing some of the HLA class II alleles and thus having a similar underlying genetic pathogenesis as $\mathrm{CD}$. Family studies will be needed to untangle such a mixture of heterogeneous IBD.

In summary, with the combination of a subclinical marker (ANCA) and molecular genetic markers (HLA-DR, -DQ alleles), genetic heterogeneity has been demonstrated within UC. There are at least two genetically distinct disorders which present with clinical features of UC: ANCA-positive UC and ANCA-negative UC, the former clearly associated with DR2, the latter likely associated with DR4. In addition, the observation that ANCA-negative UC exhibited similar magnitudes of association with the DR1 and DQ5 alleles as Crohn's disease suggests the hypothesis that a subset of ANCA-negative UC may share common genetic predispositions with $\mathrm{CD}$.

\section{Acknowledgments}

We wish to thank Andreea Muntean and Divina Magalong for technical assistance in the HLA molecular typing, and Sheila Pressman for supervising the preparation of lymphoblastoid cell lines. This work was supported by the Crohn's and Colitis Foundation of America, National Institutes of Health grants DK43026 and DK46763, the Stuart Foundations, the Cedars-Sinai Board of Governors' Chair in Medical Genetics (J. I. Rotter), and the Feintech Family Chair in Inflammatory Bowel Disease (S. R. Targan).

\section{References}

1. Yang, H., T. Shohat, and J. I. Rotter. 1992. The genetics of inflammatory bowel disease. In Inflammatory Bowel Disease. R. P. MacDermott and W. F. Stenson, editors. Elsevier Science Publishing Co., Inc., New York. pp.17-51.
2. Rotter, J. I., H. Yang, and T. Shohat. 1992. Genetic complexities of inflammatory bowel disease and its distribution among the Jewish people. In Genetic Diversity Among Jews: Diseases and Markers at the DNA Level. B. Bonne-Tamir and A. Adam, editors. Oxford University Press, New York. pp. 395-411.

3. Shanahan, F., R. H. Duerr, J. I. Rotter, H. Yang, L. R. Sutherland, C. McElree, C. J. Landers, and S. R. Targan. 1992. Neutrophil autoantibodies in ulcerative colitis: familial aggregation and genetic heterogeneity. Gastroenterology. 103:456-461.

4. Toyoda, H., S.-J. Wang, H. Yang, A. Redford, D. Magalong, D. Tyan, C. McElree, S. Pressman, F. Shanahan, S. Targan, and J. I. Rotter. 1993. Distinct associations of HLA class II genes with inflammatory bowel disease. Gastroenterology. 104:741-748.

5. van der Woude, F. J., N. Rasmussen, S. Lobatto, A. Wiik, H. Permin, L. A. van Es, M. van der Giessen, and G. K. van der Hem. 1985. Autoantibodies against neutrophils and monocytes: tool for diagnosis and marker of disease activity in Wegener's granulomatosis. Lancet. $i: 425-429$.

6. Saxon, A., F. Shanahan, C. Landers, T. Ganz, and S. R. Targan. 1990. A distinct subset of antineutrophil cytoplasmic antibodies is associated with inflammatory bowel disease. J. Allergy Clin. Immunol. 86:202-210.

7. Duerr, R. H., S. R. Targan, C. J. Landers, L. R. Sutherland, and F. Shanahan. 1991. Anti-neutrophil cytoplasmic antibodies in ulcerative colitis. Comparison with other colitides/diarrheal illnesses. Gastroenterology. 100:1590-1596.

8. Cambridge, G., D. S. Rampton, T. R. J. Stevens, D. A. McCarthy, M. Kamm, and B. Leaker. 1992. Anti-neutrophil antibodies in inflammatory bowel disease: prevalence and diagnostic role. Gut. 33:668-674.

9. Seibold, F., P. Weber, R. Klein, P. A. Berg, and K. H. Wiedmann. 1992. Clinical significance of antibodies against neutrophils in patients with inflammatory bowel disease and primary sclerosing cholangitis. Gut. 33:657-662.

10. Boyum, A. 1968. Separation of leukocytes from blood and bone marrow. Scand. J. Clin. Lab. Invest. 21:31-50.

11. Redford, A., D. Magalong, M. Onohara-Toyoda, D. Tyan, W. Riley, N. Maclaren, J. I. Rotter, and H. Toyoda. 1991. Restriction fragment length polymorphism (RFLP) heterogeneity of HLA-DQ beta genes associated with DNA fragment identical to the DR-1 beta DNA structure. Dis. Markers. 9:257-263.

12. Toyoda, H. 1992. Application of synthetic oligonucleotide probes for gene analysis. Methods Enzymol. 216:108-115.

13. Southern, E. M. 1975. Detection of specific sequence among DNA fragments separated by gel electrophoresis. J. Mol. Biol. 98:503-517.

14. Feinberg, A. P., and B. Vogelstein. 1983. A technique for radiolabelling DNA restriction endonuclease fragments to high specific activity. Anal. Biochem. 132:6-13.

15. Long, E. O., C. T. Wake, J. Gorski, and B. Mach. 1983. Complete sequence of an HLA-DR beta chain deduced from a cDNA clone and identification of multiple non-allelic DR beta chain genes. EMBO (Eur. Mol. Biol. Organ.) J. 2:389-394.

16. Larhammer, D. L., L. Schenning, K. Gustaffsson, K. Wiman, L. Claesson, L. Rask, and P. A. Peterson. 1982. Complete amino acid sequence of an HLADR antigen-like beta chain as predicted from the nucleotide sequence: Similarities with immunoglobulins and HLA-A, -B, and -C antigens. Proc. Natl. Acad. Sci. USA. 79:3687-3691.

17. Lizak, G. E., F. C. Grumet. 1980. A new micromethod for the in vitro detection of antiplatelet antibodies: C-FDA thrombocytotoxicity. Hum. Immunol. 1:87-96.

18. Snedecor, G. W., and W. G. Cochran. 1982. Statistical methods. The Iowa State University Press, Ames, IA. pp. 107-134.

19. Schlesselman, J. J. 1982. Case-Control Studies: Design, Conduct, Analysis. Oxford University Press, New York. pp. 183-190.

20. Neigut, D., R. Proujansky, M. Trucco, J. S. Dorman, S. Kocoshis, A. B. Carpenter, and E. J. Ball. 1992. Association of an HLA-DQB-1 genotype with Crohn's disease in children. Gastroenterology. 102:A671. (Abstr.).

21. Reumaux, D., L. Delecourt, J. F. Colombel, P. Duthilleul, C. GowerRousseau, L. H. Nöel, and A. Cortot. 1992. Prevalence of antineutrophil cytoplasmic auto-antibodies (ANCA) in relatives of patients with ulcerative colitis (UC). Gastroenterology. 102:A683. (Abstr.).

22. Nepom, G. T., and H. Erlich. 1991. MHC class-II molecules and autoimmunity. Annu. Rev. Immunol. 9:493-525.

23. Snook, J. A., H. J. de Silva, and D. P. Jewell. 1989. The association of autoimmune disorders with inflammatory bowel disease. Q. J. Med. (New Series) 72:835-840.

24. Tiwari, J. L., and P. I. Terasaki. 1985. HLA and Disease Associations. Springer-Verlag New York Inc., New York. pp. 32-48.

25. Sadovnick, A. D., D. W. Paty, G. Yannakoulias. 1989. Concurrence of multiple sclerosis and inflammatory bowel disease. N. Engl. J. Med. 321:762-763.

26. Rang, E. H., B. N. Brooke, J. Hermon-Taylor. 1982. Association of ulcerative colitis and multiple sclerosis. Lancet $i i: 555$.

27. Roth, M. -P., G. M. Petersen, C. McElree, C. M. Vadheim, J. F. Panish, and J. I. Rotter. 1989. Familial recurrence risk estimates of inflammatory bowel disease in Ashkenazi Jews. Gastroenterology. 96:1016-1020.

28. Yang, H., C. McElree, M.-P. Roth, F. Shanahan, S. R. Targan, and J. I. Rotter. 1992. Familial empiric risks for inflammatory bowel disease. Difference between Jews and non-Jews. Gut. In press. 